\title{
Adhesion of rough surfaces with plastic deformation
}

\section{LIXIAN ZHANG and YA-PU ZHAO*}

State Key Laboratory of Nonlinear Mechanics (LNM), Institute of Mechanics, Chinese Academy of Sciences, Beijing 100080, China

Received in final form 11 March 2004

\begin{abstract}
Adhesion is attractive and has a close relation to many modern problems of contacts between plastically deformable surfaces on the micro/nano-scale (such as microelectromechanical systems (MEMS) and nanotechnology). In the present paper a theoretical model is developed to obtain a better understanding of the adhesion of rough surfaces with plastic deformation. The present research goes beyond the Gaussian and exponential distributions of microasperity heights by introducing the description of self-affine fractal surfaces. General expressions for real contact area, total load and required separation force are derived. Two new dimensionless parameters are derived, which are used in further analysis of adhesion together with the existing roughness exponent and plastic adhesion index, giving a quantitative understanding about adhesion with plastic deformation and the relevant phenomenon.
\end{abstract}

Keywords: Adhesion; plastic deformation; self-affine fractal surface; surface roughness; distribution of microasperity heights.

\section{NOMENCLATURE}

$\begin{array}{ll}A_{\mathrm{r}} & \text { actual contact area } \\ A_{\mathrm{r}}^{\prime} & \text { real area of contact per unit area } \\ a^{\prime} & \text { contact radius of an individual microasperity } \\ a_{\mathrm{p}} & \text { contact radius during plastic loading } \\ d & \text { separation between the mean plane and the flat surface during } \\ & \text { loading } \\ E & \text { Young's modulus } \\ E^{*} & \text { effective modulus } \\ H & \text { hardness of material }\end{array}$

*To whom correspondence should be addressed. Tel.: (86-10) 6265-8008. Fax: (86-10) 6256-1284. E-mail: yzhao@lnm.imech.ac.cn 
$n$

$P$

$P^{\prime}$

$P_{\text {ad }}$

$P_{\text {ad }}^{\prime}$

$R$

$r$

$r_{\mathrm{a}}$

$U_{\mathrm{d}}$

$U_{\mathrm{p}}$

$U_{\mathrm{s}}$

$U_{\mathrm{t}}$

Y

z

$\alpha$

$\Gamma(x)$

$\Gamma(x, d)$

$\Delta \gamma$

$\gamma_{i}(i=1,2)$

$\gamma_{12}$

$\delta$

$\zeta$

$\zeta_{\alpha}$

$\eta$

$\theta$

$\theta_{\alpha}$

$\lambda$

$\mu$

$v$

$\xi$ number of asperities per unit area of rough surface

number of asperities per unit area that are able to make contact force that pressed surfaces together

load per unit area to compress the microasperities

adhesion force, also separation force

adhesion force per unit area

equivalent radius of smooth sphere

radius of a perfectly plastic hemispherical tip

radius of curvature of an asperity

energy absorbed in plastic deformation of a perfectly plastic hemispherical tip

work done by the applied load

contribution due to work of adhesion

total energy of system

yield stress of material

peak height above the mean line

roughness exponent

gamma function, $\Gamma(x)=\int_{0}^{\infty} e^{-t} t^{x-1} \mathrm{~d} t$

incomplete gamma function, $\Gamma(x, d)=\int_{d}^{\infty} e^{-t} t^{x-1} \mathrm{~d} t$

Dupré adhesion or work of adhesion

surface energy of material

interface energy of contacting materials 1 and 2

vertical displacement of a perfectly plastic hemispherical tip

length scale for the Gaussian distributed rough surface

length scale of the fractal surface

ratio of the separation between the mean plane and the flat surface during loading to the standard deviation of the peak heights

adhesion parameter for Gaussian distributed rough surface

generalized adhesion parameter for a self-affine fractal surface

plastic adhesion index

ratio of the work of adhesion to the product of the standard deviation of the peak heights and the hardness

Poisson's ratio

correlation length of the self-affine fractal surface parallel to the surface

standard deviation of peak heights 


\section{INTRODUCTION}

The interaction between deformable solids, which is derived from physical attractive and repulsive forces, has a close relevance to contact problem, friction and wear of contacting surfaces $[1,2]$. Larger-scale systems are more influenced by inertia effects, while smaller-scale systems are more influenced by surface effects. In microelectromechanical systems (MEMS) and nanotechnology fields, the adhesion becomes more significant [3]. Developing a sophisticated understanding of adhesion between solid-solid surfaces will help controlling processes such as bonding and debonding [4] that are essential to fabrication of MEMS. It has been known for a long time that the surface roughness is very important in determining the interaction force between the contacting surfaces. Since the actual contacting surfaces are rough, the effect of the roughness will lead to the scenario that the actual contact area occurs at the peaks of the inevitable surface irregularities where the local contact pressure is very high. Furthermore, the details of the distribution of asperities can also affect the adhesion.

Greenwood and Williamson, assuming the asperity heights distribution to be Gaussian, discovered that many important properties of the contact were almost independent of the details of the local asperity behavior [5]. A dimensionless parameter, plasticity index, was proposed in Ref. [5] to predict the plastic deformation of asperities. Johnson et al. [6] studied the adhesion between two elastic spheres with radii $R_{1}$ and $R_{2}$, and concluded that the adhesion force or force required to separate the bodies $P_{\text {ad }}$, was independent of both the applied load and the elastic constants of the materials, i.e.

$$
P_{\text {ad }}=-\frac{3}{2} \pi \Delta \gamma R
$$

where $R=R_{1} R_{2} /\left(R_{1}+R_{2}\right)$ is the equivalent radius, and $\Delta \gamma=\gamma_{1}+\gamma_{2}-\gamma_{12}$ is the Dupré adhesion or work of adhesion, with $\gamma_{1}$ and $\gamma_{2}$ being the surface energies of the two spheres and $\gamma_{12}$ being the interface energy. It should be noted that there is no consistent term for $P_{\text {ad }}$ in equation (1) in the literature, separation force and pull-off force are also used [7]. Subsequently, Fuller and Tabor [8] investigated the effect of surface roughness with Gaussian distribution on the adhesion of elastic solids and introduced an adhesion parameter $\theta$ given by

$$
\theta=\frac{E \sigma^{3 / 2}}{r_{\mathrm{a}}^{1 / 2} \Delta \gamma},
$$

where $E$ is Young's modulus, $\sigma$ the standard deviation of the peak heights and $r_{\mathrm{a}}$ the radius of curvature of an asperity. This dimensionless parameter represents the relative importance of surface roughness and adhesion, and is the ratio between the elastic energy and the work of adhesion, assuming that complete contact has occurred. When $\theta \gg 1$ only partial contact occurs, where the elastic solids make contact only at the tops of the highest asperities, while a complete contact occurs when $\theta \ll 1$. 
It was during the 1950s that experiments showed plastic deformation of particles caused by adhesion forces [9]. Krupp [10] originally proposed that the adhesion forces could rise to such large values that they would exceed the elastic limit of the material and induce irreversible, plastic flow. Experiments on gold spheres were carried out and showed that only small particles exhibited plastic deformation due to adhesion force. Easterling and Tholen [11] and Tholen [12] showed plastic deformation in electron micrographs of metal particles sticking together. They observed dislocations and plastic twins emanating from the highly stressed contact region. Scanning electron microscope (SEM) studies were performed on polystyrene spheres sitting on polished silicon surfaces by Rimai et al. [13], and plastic deformation was observed due to adhesion forces.

Johnson [14] derived both elastic adhesion index and plastic adhesion index. The latter is given as

$$
\lambda=\frac{\pi^{2} H^{4} r_{\mathrm{a}} \sigma}{8 \Delta \gamma^{2} E^{* 2}},
$$

where $H$ is the material hardness, and

$$
E^{*}=\left(\frac{1-v_{1}^{2}}{E_{1}}+\frac{1-v_{2}^{2}}{E_{2}}\right)^{-1}
$$

is the effective modulus, where $E_{1,2}$ and $v_{1,2}$ are Young's moduli and Poisson's ratios of the two bodies, respectively. According to the von Mises flow rule, the hardness, $H$, is related to yield stress, $Y$, by the Tabor relation [15]: $H=3 Y$. Also, Johnson investigated the adhesion problem for both elastic and plastic deformations assuming an exponential distribution of asperity heights. For plastic deformation, the ratio of the force that separated the surfaces, $P_{\text {ad }}$, to the force that pressed them together, $P$, was obtained as

$$
\frac{P_{\mathrm{ad}}}{P}=-1+\frac{1+\lambda}{\lambda} \exp \left(-\frac{1}{\lambda}\right)
$$

With the same assumed heights distribution Chowdhury and Pollock [16] studied adhesion between metal surfaces with plastic deformation. Considering multiasperities, the total real area of contact per unit area is given as

$$
A_{\mathrm{r}}^{\prime}=2 \pi n r_{\mathrm{a}} \sigma,
$$

where $n$ denotes the number of asperities per unit contact area. And the adhesion coefficient or ratio of total adhesion force to the total compression force is expressed as

$$
\frac{P_{\mathrm{ad}}}{P}=\frac{1}{1-\Delta \gamma /(H \sigma)}\left\{-1+\frac{1+\lambda}{\lambda} \exp \left(-\frac{1}{\lambda}\right)\right\} .
$$

For plastic adhesion index $\lambda>2$, very small adhesion is predicted [14].

Recently, considering the influence of the microstructure of self-affine fractal surfaces, Chow studied the adhesion between elastic deformable fractal surfaces 
$[17,18]$. Persson studied the contact mechanics between solids on all roughness length scales, discussed the relation of adhesion parameter to different roughness length scales $[19,20]$ and derived the generalized adhesion parameter $\theta_{\alpha}$ for a selfaffine fractal surface as

$$
\theta_{\alpha}=\theta\left(\frac{\zeta_{\alpha}}{\zeta}\right)^{2 \alpha-1},
$$

where $\theta$ and $\zeta$ are the adhesion parameter and length scale for the Gaussiandistributed rough surface, respectively, $\alpha$ is the dimension of the self-affine fractal surface and $0<\alpha<1, \zeta_{\alpha}$ is the length scale of the fractal surface. It should be noted that the generalized adhesion parameter in equation (7) is reduced to the adhesion parameter for Gaussian distribution in equation (2) when $\alpha=1 / 2$. It should be noted that the generalized adhesion parameter in equation (7) is valid for surface roughness on a wide distribution of length scales.

Under ideal equilibrium conditions, the work of adhesion is considered to be a well-defined reversible thermodynamic quantity, i.e. the work done on bringing two unit areas of surfaces together and the work needed to separate two unit areas of surfaces from contact are the same. But under most realistic conditions there is adhesion hysteresis: the work needed to separate two unit areas of surfaces is always greater than that originally gained on bringing them together; in other words, the approach/separation cycles are thermodynamically irreversible [21] and, therefore, energy is dissipated. Adhesion contact with plastic deformation is one of the mechanisms of adhesion hysteresis [21, 22]. The research on adhesion in case of plastic deformation has been in the past mainly restricted to the Gaussian or exponential distribution of asperity heights. The present research studied the adhesion of rough surfaces with plastic deformation. Furthermore, the effects of relevant parameters, such as roughness exponent, plastic adhesion index and two new dimensionless terms introduced in this theoretical model are analyzed.

\section{PLASTIC DEFORMATION THEORY OF A SINGLE ASPERITY}

Contact happens between surfaces of two bodies when they are pressed together. When the applied load exceeds a critical value, an irreversible curve will be obtained in the loading-unloading experiment, which indicates that the applied load has compressed the microasperity plastically. When a perfect plastic contact due to adhesion is assumed, i.e. when a perfectly plastic hemispherical tip of radius $r$ is pressed against a perfectly horizontal rigid plane, the vertical displacement $\delta$ is given by the geometric relationship as [16]:

$$
a_{\mathrm{p}}^{2} \approx 2 r \delta,
$$

where $a_{\mathrm{p}}$ represents the contact radius during plastic loading, and equation (8) holds provided that the change in contact geometry due to the material flow is 
neglected [16]. The work done by the applied load, $U_{\mathrm{p}}$, is given as [16]

$$
U_{\mathrm{p}}=-P \delta \text {. }
$$

The energy absorbed in plastic deformation, $U_{\mathrm{d}}$, is given by

$$
U_{\mathrm{d}}=\int_{0}^{\delta} \pi a_{\mathrm{p}}^{2} H \mathrm{~d} \delta,
$$

and the contribution due to the work of adhesion is

$$
U_{\mathrm{s}}=-\pi a_{\mathrm{p}}^{2} \Delta \gamma
$$

Thus the total energy of this system is obtained as

$$
U_{\mathrm{t}}=U_{\mathrm{p}}+U_{\mathrm{d}}+U_{\mathrm{s}} \text {. }
$$

Considering the energy equilibrium condition $\partial U_{\mathrm{t}} / \partial \delta=0$, one has

$$
P=\pi a_{\mathrm{p}}^{2} H-2 \pi r \Delta \gamma .
$$

Without the effect of work of adhesion, the applied load is

$$
P=\pi a_{\mathrm{p}}^{2} H
$$

which is adopted in Johnson's analysis of adhesion in case of perfectly plastic deformation [14]. Therefore, if a single area of contact is assumed, the contact size can be derived from the resistance measurements by varying the applied load [16], and values of both $\Delta \gamma$ and $H$ can be derived from equation (13).

During unloading, adhesion forces play an important role. Adhesion contact established during loading is capable of appreciable plastic extension before fracture, especially for materials with high modulus and low hardness. If we take into account the condition that plastic deformation of the material during loading is followed by ductile extension of the contact, then a criterion for ductile extension is given by [14]

$$
H^{2}<4 \frac{\Delta \gamma E^{*}}{\pi a_{\mathrm{p}}}
$$

Here it is assumed that the tip of microasperity will extend in a ductile manner when the contact pressure just exceeds the hardness $H$ of the material during unloading. Generally, the softer the material, the greater the possibility of plastic extension before fracture [14].

\section{THEORETICAL MODEL OF ADHESION OF ROUGH SURFACES WITH PLASTIC DEFORMATION}

A theoretical formulation of applied load by assuming a single area of contact was shown above, but in fact the real surfaces at the nano/micro-level are rough. The surface profile of single crystal silicon $(8 \mu \mathrm{m} \times 8 \mu \mathrm{m})$ obtained using an atomic 
force microscope (AFM) is shown in Fig. 1. Figure 1 shows that a multi-asperity structure at microscopic scale exists in the real surface. For simplicity and without loss of generality, we consider a randomly rough deformable surface and a perfectly rigid smooth surface in contact as depicted in Fig. 2 [23], where the separation between the mean plane and the flat surface during loading is $d$, the asperity peaks are assumed to have a radius of curvature $r_{\mathrm{a}}$ and the peak height above the mean line is $z$.

Fractals present a natural language for describing the scaling behavior of roughness on all length scales [18]. Self-affine fractals are invariant under an anisotropic dilation. A general distribution of microasperity heights derived from the description of fractal surfaces is introduced into the present analysis of adhesion. The distribution of asperity heights is expressed as [17]:

$$
\Psi(z)=\Psi_{0} \exp \left[-\alpha\left(\frac{z}{\sigma}\right)^{1 / \alpha}\right], \quad 0<\alpha \leqslant 1,
$$

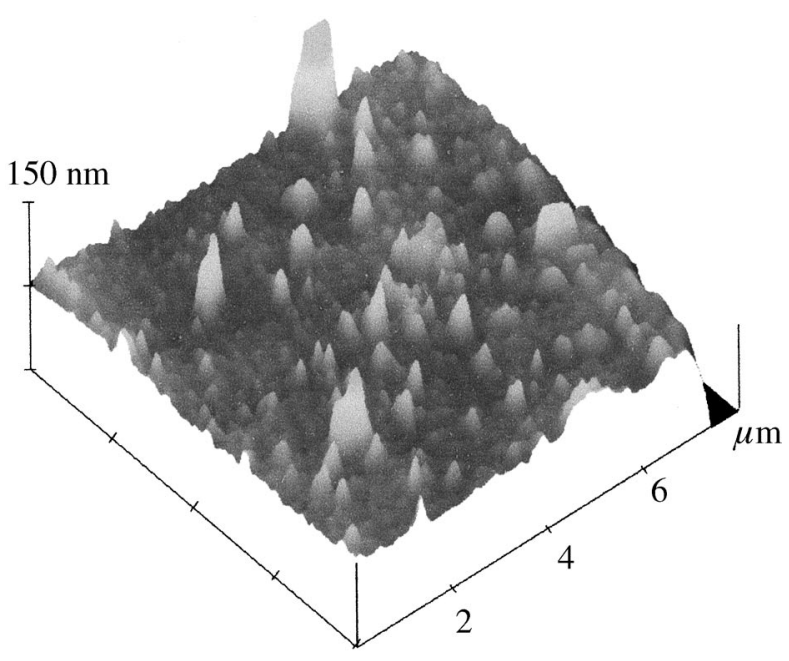

Figure 1. Surface roughness profile of single crystal silicon $(8 \mu \mathrm{m} \times 8 \mu \mathrm{m})$ obtained by an atomic force microscope (AFM).

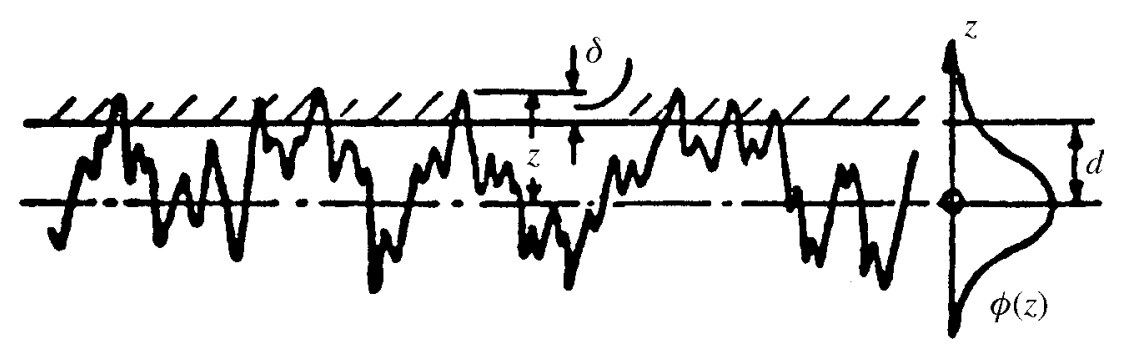

Figure 2. Contact of a random rough deformable surface with a rigid plane. 
where

$$
\Psi_{0}=\left[\sigma \int_{0}^{\infty} \exp \left(-\alpha u^{1 / \alpha}\right) \mathrm{d} u\right]^{-1} \frac{1}{\sigma \alpha^{1-\alpha} \Gamma(\alpha)},
$$

where $\Gamma$ is the gamma function and is defined as $\Gamma(x)=\int_{0}^{\infty} \mathrm{e}^{-t} t^{x-1} \mathrm{~d} t, \sigma$ is the standard deviation of the peak asperity heights and $\alpha$ is the roughness exponent which can be calculated using digital data obtained from AFM [24]. For a given domain size of short-range surface profile, the smaller the exponent $\alpha$, the rougher the local variation of the surface structure, and smoother hills and valleys are expected as $\alpha$ approaches 1 [25]. When $\alpha=1 / 2$, we obtain the Gaussian distribution function from the above two equations, and when $\alpha=1$, we obtain the exponential distribution function. The radius of curvature of asperities $r_{\mathrm{a}}$ is given as [17]:

$$
\frac{1}{r_{\mathrm{a}}}=\frac{2 \sigma}{\xi^{2}},
$$

where $\xi$ is the correlation length of the self-affine fractal surface parallel to the surface. The vertical deformation $\delta$ is defined as

$$
\delta=z-d .
$$

If the rough surface has $N$ asperities per unit area, the number of asperities, $n$, per unit area that are able to make contact is given by

$$
n=N \int_{d}^{\infty} \Psi(z) \mathrm{d} z=N \Gamma^{-1}(\alpha) \Gamma\left(\alpha, \alpha(d / \sigma)^{1 / \alpha}\right),
$$

where the two-argument gamma function $\Gamma(x, d)$ is also called incomplete gamma function and is defined as $\Gamma(x, d)=\int_{d}^{\infty} \mathrm{e}^{-t} t^{x-1} \mathrm{~d} t$. As shown by Archard [26], the quantities $r_{\mathrm{a}}, \sigma$ and $N$ are not independent of each other but are related, i.e. $r_{\mathrm{a}} \sigma N=$ constant. The value of this constant is between 0.05 and 0.1 [4].

The total real area of contact per unit area is given by [16]

$$
A_{\mathrm{r}}^{\prime}=N \int_{d}^{\infty} \pi a^{\prime 2} \Psi(z) \mathrm{d} z
$$

where $a^{\prime}$ is the contact radius of an individual microasperity. Replacing the contact radius of a microasperity by $\left(2 r_{\mathrm{a}} \delta\right)^{1 / 2}$ derived simply from geometric considerations and integrating the former expression, one has

$$
A_{\mathrm{r}}^{\prime}=2 \pi n r_{\mathrm{a}} \sigma \alpha^{-\alpha}\left[\frac{\Gamma\left(2 \alpha, \alpha(d / \sigma)^{1 / \alpha}\right)}{\Gamma\left(\alpha, \alpha(d / \sigma)^{1 / \alpha}\right)}-\frac{\alpha^{\alpha} d}{\sigma}\right] .
$$

For a roughness exponent of 1 , this equation is reduced to equation (5). Considering the microasperities that are deformed plastically at an applied load, the total load per 
unit area to compress the microasperities is given by

$$
P^{\prime}=N \int_{d}^{\infty}\left(\pi a^{\prime 2} H-2 \pi r_{\mathrm{a}} \Delta \gamma\right) \Psi(z) \mathrm{d} z
$$

Similarly substituting the contact radius of a microasperity by $\left(2 r_{\mathrm{a}} \delta\right)^{1 / 2}$, we have

$$
P^{\prime}=2 \pi n r_{\mathrm{a}} H \sigma \alpha^{-\alpha}\left[\frac{\Gamma\left(2 \alpha, \alpha(d / \sigma)^{1 / \alpha}\right)}{\Gamma\left(\alpha, \alpha(d / \sigma)^{1 / \alpha}\right)}-\frac{\alpha^{\alpha} d}{\sigma}\right]-2 \pi n r_{\mathrm{a}} \Delta \gamma .
$$

Combining equation (24) with equation (22) gives

$$
P=A_{\mathrm{r}} \frac{H\left[\sigma \Gamma\left(2 \alpha, \alpha(d / \sigma)^{1 / \alpha}\right)-\alpha^{\alpha} d \Gamma\left(\alpha, \alpha(d / \sigma)^{1 / \alpha}\right)\right]-\Delta \gamma \alpha^{\alpha} \Gamma\left(\alpha, \alpha(d / \sigma)^{1 / \alpha}\right)}{\sigma \Gamma\left(2 \alpha, \alpha(d / \sigma)^{1 / \alpha}\right)-d \alpha^{\alpha} \Gamma\left(\alpha, \alpha(d / \sigma)^{1 / \alpha}\right)},
$$

where $A_{\mathrm{r}}$ is the actual contact area and $P$ the real applied load.

From equation (25) we make an interesting observation that the microasperities can be plastically deformed, even at zero load, when the following condition is satisfied:

$$
\frac{H\left[\sigma \Gamma\left(2 \alpha, \alpha(d / \sigma)^{1 / \alpha}\right)-\alpha^{\alpha} d \Gamma\left(\alpha, \alpha(d / \sigma)^{1 / \alpha}\right)\right]-\Delta \gamma \alpha^{\alpha} \Gamma\left(\alpha, \alpha(d / \sigma)^{1 / \alpha}\right)}{\sigma \Gamma\left(2 \alpha, \alpha(d / \sigma)^{1 / \alpha}\right)-d \alpha^{\alpha} \Gamma\left(\alpha, \alpha(d / \sigma)^{1 / \alpha}\right)}=0 .
$$

The above equation can be rewritten as follows:

$$
\frac{\Delta \gamma}{H}=\frac{\sigma \Gamma\left(2 \alpha, \alpha(d / \sigma)^{1 / \alpha}\right)-\alpha^{\alpha} d \Gamma\left(\alpha, \alpha(d / \sigma)^{1 / \alpha}\right)}{\alpha^{\alpha} \Gamma\left(\alpha, \alpha(d / \sigma)^{1 / \alpha}\right)} .
$$

If the roughness exponent is taken as $1(\alpha=1)$, viz., the distribution of microasperity heights conforms to exponential distribution, equations (24) and (27) can be rewritten as

$$
P^{\prime}=2 \pi n r_{\mathrm{a}} \sigma[H-(\Delta \gamma / \sigma)]
$$

and

$$
\sigma=\frac{\Delta \gamma}{H}
$$

which are the same as equations (12) and (14) in Ref. [16], respectively.

The force required to separate the contacting surfaces during ductile extension, or the adhesion force, can be determined by considering those asperities that are plastically deformed in such an extension. Only those microasperities that satisfy the condition of ductile extension described as equation (15) would extend in a ductile manner. Considering the criterion of ductile extension, we can rewrite the condition of equation (15) as $\delta<\delta_{\mathrm{a}} \equiv 8 \Delta \gamma^{2} E^{* 2} /\left(\pi^{2} r_{\mathrm{a}} H^{4}\right)$. Then Johnson's plastic adhesion index, $\lambda$, can be formulated as $\lambda \equiv \sigma / \delta_{\mathrm{a}}$ which is the same as equation (3). The adhesion force per unit area is given by

$$
P_{\mathrm{ad}}^{\prime}=N \int_{d}^{d+\delta_{\mathrm{a}}} H \pi a^{\prime 2} \Psi(z) \mathrm{d} z
$$


And integration of the above equation yields

$$
\begin{aligned}
P_{\mathrm{ad}}^{\prime}= & 2 \pi n H r \alpha^{-\alpha} \sigma \Gamma^{-1}\left(\alpha, \alpha\left(\frac{d}{\sigma}\right)^{1 / \alpha}\right)\left[\Gamma\left(2 \alpha, \alpha\left(\frac{d}{\sigma}\right)^{1 / \alpha}\right)\right. \\
& -\Gamma\left(2 \alpha, \alpha\left(\frac{\delta_{\mathrm{a}}}{\sigma}+\frac{d}{\sigma}\right)^{1 / \alpha}\right)-\frac{\alpha^{\alpha} d}{\sigma} \Gamma\left(\alpha, \alpha\left(\frac{d}{\sigma}\right)^{1 / \alpha}\right) \\
& \left.+\frac{\alpha^{\alpha} d}{\sigma} \Gamma\left(\alpha, \alpha\left(\frac{\delta_{\mathrm{a}}}{\sigma}+\frac{d}{\sigma}\right)^{1 / \alpha}\right)\right] .
\end{aligned}
$$

\section{DISCUSSION}

Considering the expressions derived above, in addition to the plastic adhesion index $\lambda$ and the roughness exponent $\alpha$, there are two new dimensionless terms, $\Delta \gamma /(\sigma H)$ and $d / \sigma$, which play important roles in the adhesion of rough surfaces with plastic deformation. Therefore, two dimensionless parameters are introduced as

$$
\mu \equiv \frac{\Delta \gamma}{\sigma H},
$$

and

$$
\eta \equiv \frac{d}{\sigma}
$$

where $\mu$ is the ratio of the work of adhesion to the product of the standard deviation of the peak heights and the hardness. The larger the $\mu$, the easier the plastic deformation becomes. The dimensionless parameter $\eta$ is the ratio of the separation between the mean plane and the flat surface during loading to the standard deviation of the peak heights. Considering equations (27) and (29), one can see that $\mu=1$ represents the condition of plastic deformation at zero load for exponential distribution of asperity heights.

Using these dimensionless parameters, equation (22) is rewritten as

$$
\frac{A_{\mathrm{r}}^{\prime}}{2 \pi n r_{\mathrm{a}} \sigma}=\alpha^{-\alpha} \frac{\Gamma\left(2 \alpha, \alpha \eta^{1 / \alpha}\right)}{\Gamma\left(\alpha, \alpha \eta^{1 / \alpha}\right)}-\eta .
$$

The term on the left-hand side of the above equation is normalized by $2 \pi n r_{\mathrm{a}} \sigma$ which is the actual contact area per unit area for exponential distribution of asperity heights. The influence of the parameters $\alpha$ and $\eta$ on the dimensionless form of real contact area is shown in Fig. 3. Figure 3 illustrates that the real contact area is directly proportional to the roughness exponent, i.e. the rougher the surface, the smaller the real contact area. However, the actual contact area decreases with the increment of the parameter $\eta$.

Equation (24) is rewritten as

$$
\frac{P^{\prime}}{2 \pi n r_{\mathrm{a}} \sigma H}=\alpha^{-\alpha} \frac{\Gamma\left(2 \alpha, \alpha \eta^{1 / \alpha}\right)}{\Gamma\left(\alpha, \alpha \eta^{1 / \alpha}\right)}-\eta-\mu,
$$




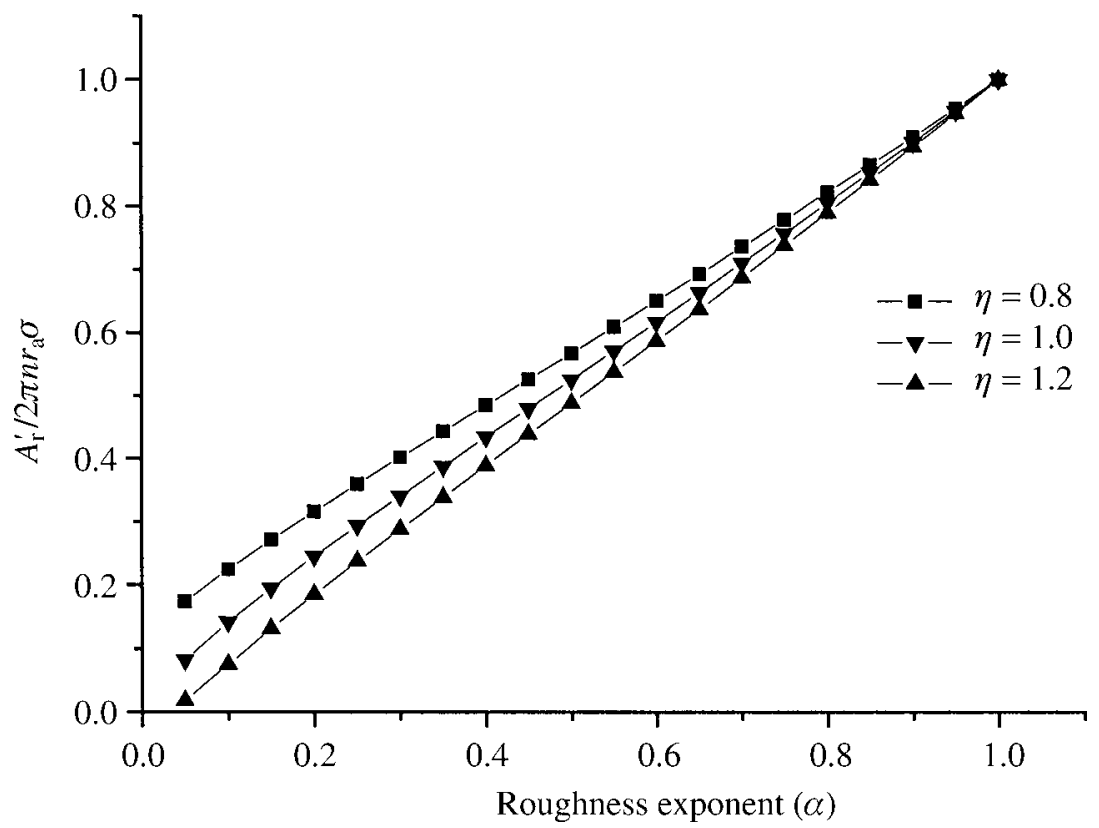

Figure 3. Variation of the dimensionless term $A_{\mathrm{r}}^{\prime} / 2 \pi n r_{\mathrm{a}} \sigma$ with roughness exponent $\alpha$ for $\eta=0.8$, 1.0 and 1.2 .

and similarly the influence of $\alpha, \mu$ and $\eta$ on the ratio $P^{\prime} / 2 \pi n r_{\mathrm{a}} \sigma H$ is shown in Fig. 4. The dimensionless load is directly proportional to the roughness exponent $\alpha$; in addition, both smaller parameter $\eta$ and smaller parameter $\mu$ correspond to a larger value of the dimensionless load. An interesting phenomenon is observed that the dimensionless load term becomes negative in a certain $\alpha$ range, for example, when $\alpha$ approaches zero, $\eta$ equals 1.2 and $\mu$ equals 0.1 .

By using equation (32), equation (27) is rewritten as

$$
\mu=\alpha^{-\alpha} \frac{\Gamma\left(2 \alpha, \alpha \eta^{1 / \alpha}\right)}{\Gamma\left(\alpha, \alpha \eta^{1 / \alpha}\right)}-\eta,
$$

and the influence of $\alpha$ and $\eta$ on the required condition of plastic deformation at zero load is shown in Fig. 5. One can see that the parameter $\mu$ is directly proportional to the roughness exponent $\alpha$, but it is inversely proportional to the parameter $\eta$. When $\alpha=1$, the condition is the same as equation (29), i.e. $\mu$ equals 1 and is independent of the parameter $\eta$. The parameter $\mu$ decreases with decreasing $\alpha$. For $\alpha<1$, a smaller $\eta$ corresponds to a larger $\mu$.

Considering equations (24) and (31), the ratio between the force to separate the contacting surfaces and the force that presses them together is expressed as

$$
\frac{P_{\mathrm{ad}}^{\prime}}{P^{\prime}}=\frac{\Gamma\left(2 \alpha, \alpha \eta^{1 / \alpha}\right)-\Gamma\left(2 \alpha, \alpha(1 / \lambda+\eta)^{1 / \alpha}\right)-\alpha^{\alpha} \eta \Gamma\left(\alpha, \alpha \eta^{1 / \alpha}\right)+\alpha^{\alpha} \eta \Gamma\left(\alpha, \alpha(1 / \lambda+\eta)^{1 / \alpha}\right)}{\Gamma\left(2 \alpha, \alpha \eta^{1 / \alpha}\right)-\alpha^{\alpha} \eta \Gamma\left(\alpha, \alpha \eta^{1 / \alpha}\right)-\mu \alpha^{\alpha} \Gamma\left(\alpha, \alpha \eta^{1 / \alpha}\right)},
$$




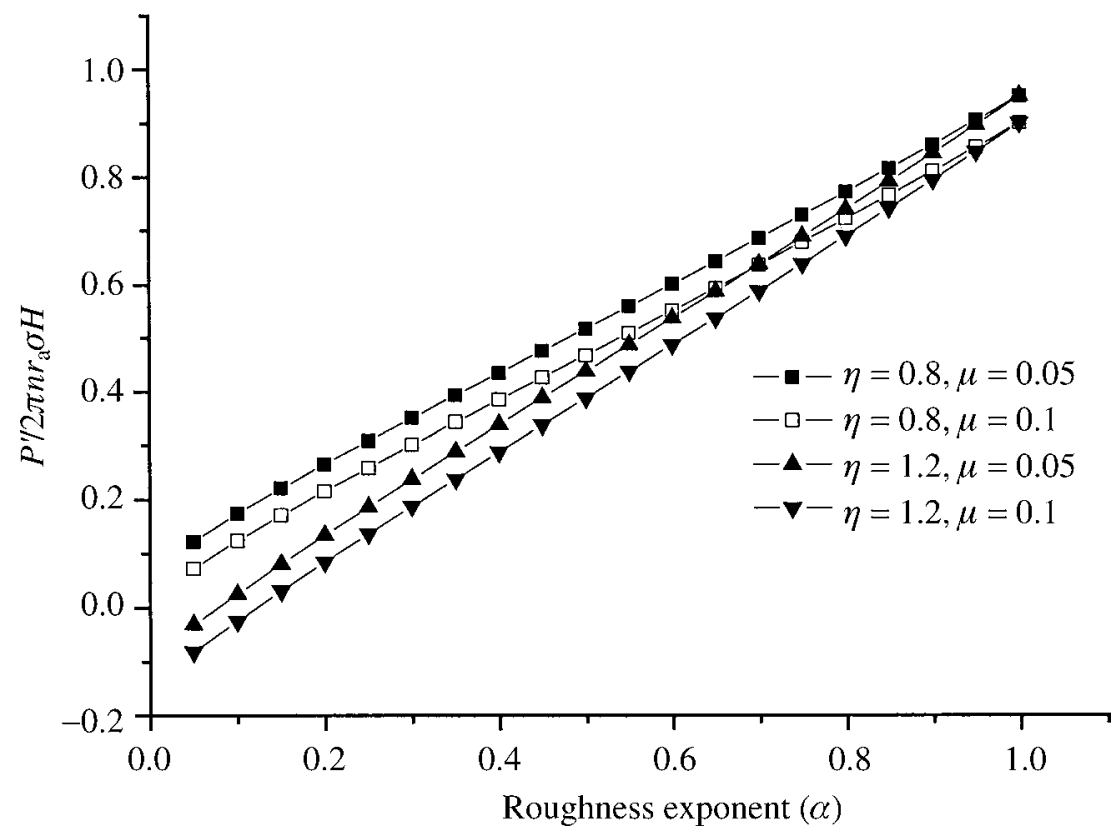

Figure 4. Variation of the dimensionless term $P^{\prime} / 2 \pi n r_{\mathrm{a}} \sigma H$ with roughness exponent $\alpha$ for various $\eta$ and $\mu$.

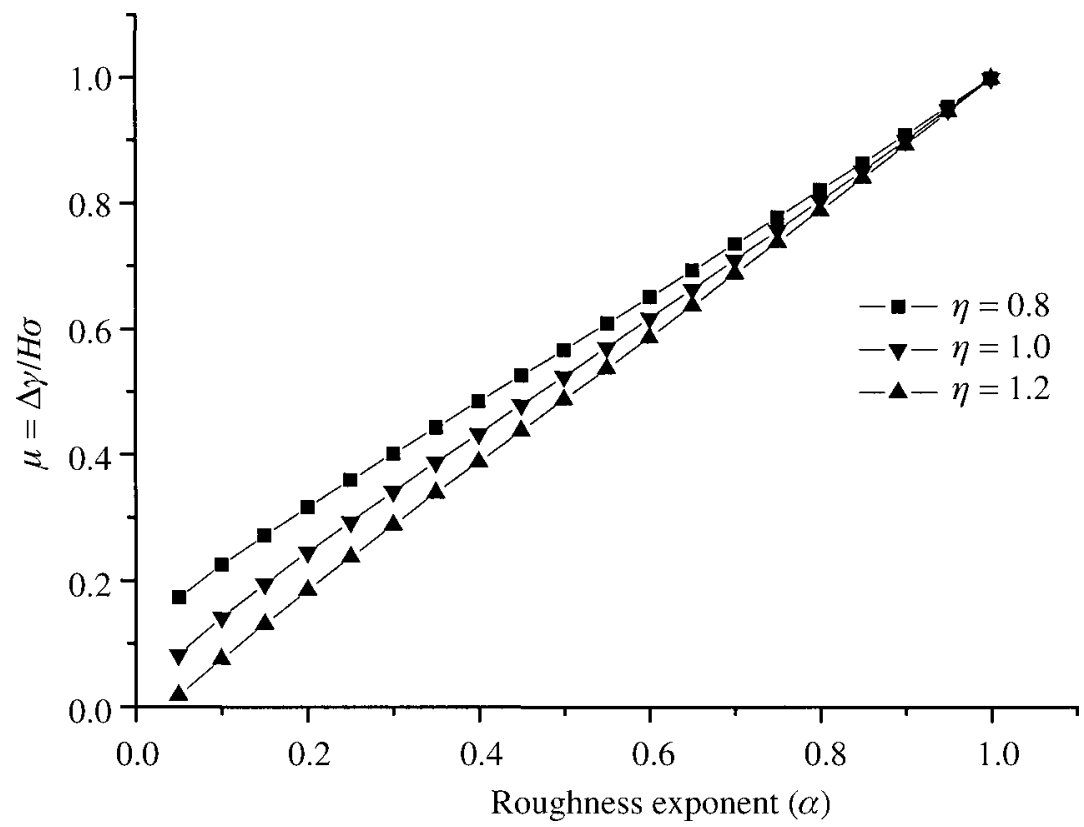

Figure 5. Variation of $\mu=\Delta \gamma /(H \sigma)$ with roughness exponent $\alpha$ for $\eta=0.8,1.0$ and 1.2 which represents the condition for plastic deformation at zero load. 


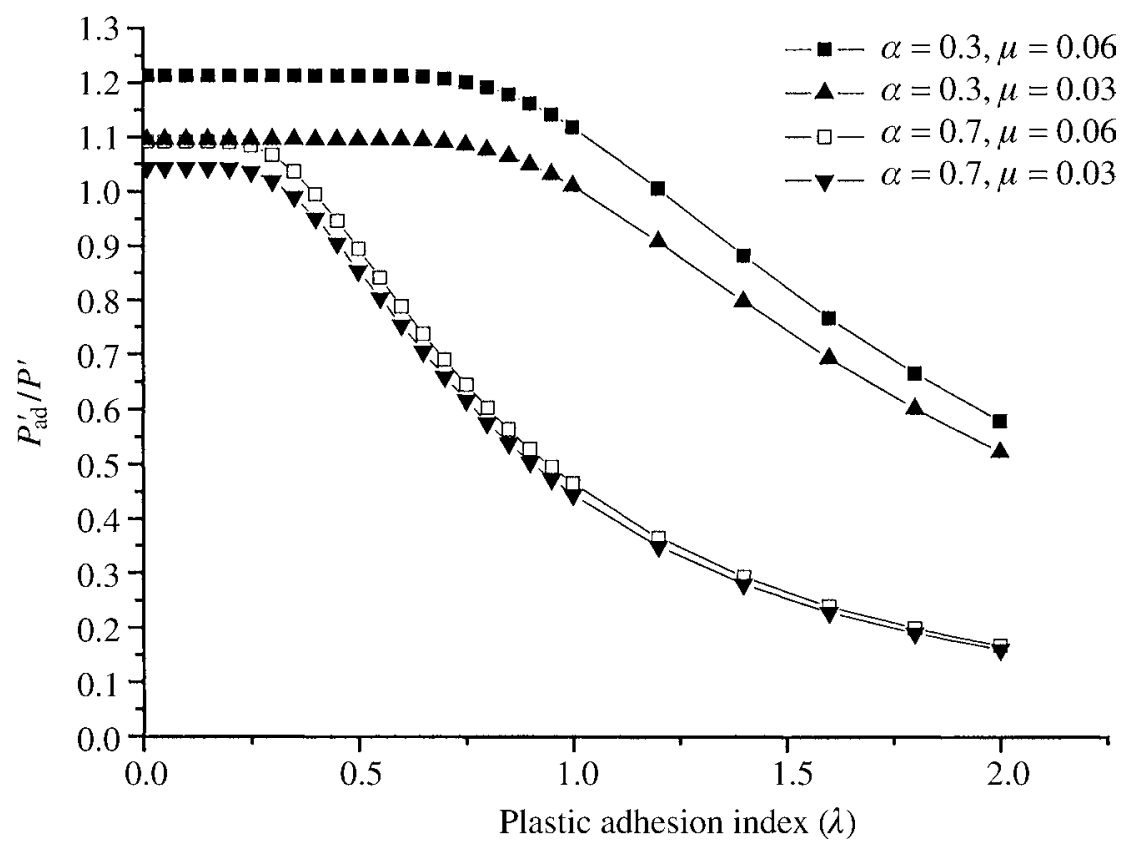

Figure 6. Variation of ratio of adhesion force to total load with plastic adhesion index $\lambda$ for $\eta=1.0$ and various $\alpha$ and $\mu$.

which is shown in Figs 6 and 7 as functions of the plastic adhesion index $\lambda$ for various $\alpha, \eta$ and $\mu$. If the roughness exponent is taken as 1 , equation (37) can be reduced to equation (6). These figures show that the ratio $P_{\mathrm{ad}}^{\prime} / P^{\prime}$ is inversely proportional to both the plastic adhesion index $\lambda$ and to the roughness exponent $\alpha$. The ratio is approximately constant when $\lambda$ is less than a limiting value which is sensitive to $\alpha$. For example, this limiting value is about 0.25 for $\alpha=0.7$ and about 0.75 for $\alpha=0.3$. Then it drops rapidly with increasing $\lambda$. It is seen from Fig. 6 that for constant $\alpha$, a larger $\mu$ corresponds to a larger $P_{\mathrm{ad}}^{\prime} / P^{\prime}$ ratio. It is also seen from Fig. 7 that a larger $\eta$ corresponds to a larger $P_{\text {ad }}^{\prime} / P^{\prime}$ ratio. An unusual phenomenon appears that the upper limit of the ratio $P_{\mathrm{ad}}^{\prime} / P^{\prime}$ is more than unity. With the present assumption of perfectly plastic adhesion contact, the effect of the work of adhesion explains this behavior.

\section{CONCLUSIONS}

A theoretical model is developed to describe the adhesion between plasticallydeformable fractal surfaces whose asperity heights conform to a general distribution. General expressions for real contact area, total load and the required separation force are obtained. In special cases, for $\alpha=1 / 2$, the results obtained in this study can be reduced to those of Gaussian distribution of surface asperities and for $\alpha=1$ they can be reduced to those of exponential distribution of surface asperities. 


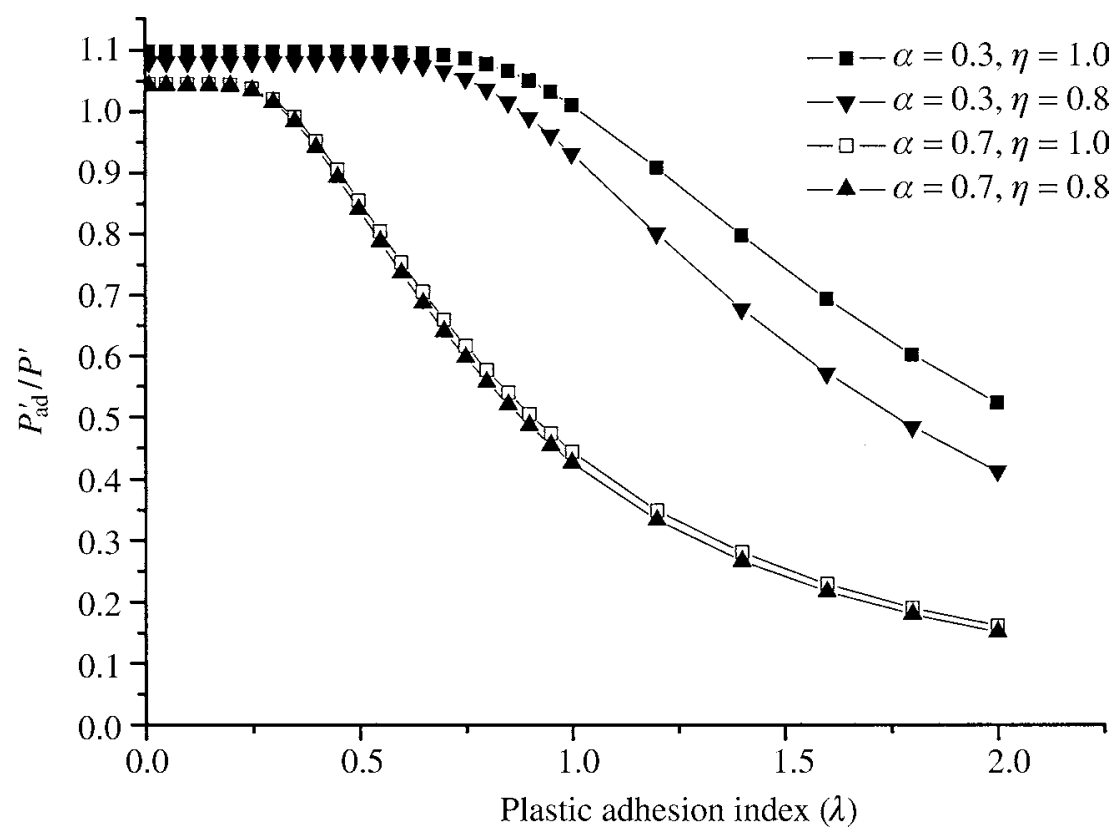

Figure 7. Variation of ratio of adhesion force to total load with plastic adhesion index $\lambda$ for $\mu=0.03$ and various $\alpha$ and $\eta$.

The condition has been determined for interface plastic deformation due to the work of adhesion at zero external load for asperities with fractal distribution. For exponential distribution $(\alpha=1)$, this condition reduces to $\mu=1$, or equivalently $\sigma=\Delta \gamma / H[16]$.

In addition to the roughness exponent $\alpha$ and the plastic adhesion index $\lambda$, two new dimensionless parameters, $\eta=d / \sigma$ and $\mu=\Delta \gamma /(\sigma H)$, are derived. The influences of these dimensionless parameters on adhesion with plastic deformation are studied.

\section{Acknowledgements}

This research was supported by the Distinguished Young Scholar Fund of the National Natural Science Foundation of China (NSFC) (Grant No. 10225209 and No. 90305020), key project from the Chinese Academy of Sciences (Grant No. KJCX2-SW-L2) and NSFC-RGC Joint Project (Grant No. 50131160739).

\section{REFERENCES}

1. D. Tabor, in: Surface Physics of Materials, J. M. Blakely (Ed.), Volume 2, Chapter 10. Academic Press, New York, NY (1975).

2. W. C. Wake, Adhesion and the Formulation of Adhesives, 2nd edn. Applied Science, London (1982).

3. Y. P. Zhao, L. S. Wang and T. X. Yu, J. Adhesion Sci. Technol. 17, 519 (2003). 
4. C. Gui, M. Elwenspoek, N. Tas and J. G. E. Gardeniers, J. Appl. Phys. 85, 7448 (1999).

5. J. A. Greenwood and J. B. P. Williamson, Proc. R. Soc. Lond. A295, 300 (1966).

6. K. L. Johnson, K. Kendall and A. D. Roberts, Proc. R. Soc. Lond. A324, 301 (1971).

7. X. H. Shi and Y. P. Zhao, J. Adhesion Sci. Technol. 18, 55 (2004).

8. K. N. G. Fuller and D. Tabor, Proc. R. Soc. Lond. A345, 327 (1975).

9. K. Kendall, Molecular Adhesion and Its Applications. Kluwer Academic/Plenum Publishers, New York, NY (2001).

10. E. Krupp, Adv. Colloid Interface Sci. 1, 111 (1967).

11. K. E. Easterling and A. R. Tholen, Acta Metall. 20,1001 (1972).

12. A. R. Tholen, in: Microscopic Aspects of Adhesion and Lubrication, J. M. Georges (Ed.), p. 263. Elsevier, Amsterdam (1982).

13. D. S. Rimai, L. P. DeMejo and R. C. Bowen, in: Fundamentals of Adhesion and Interfaces, D. S. Rimai, L. P. DeMejo and K. L. Mittal (Eds), p. 1. VSP, Utrecht (1995).

14. K. L. Johnson, in: Theoretical and Applied Mechanics, W. T. Koiter (Ed.), p.133. North-Holland, Amsterdam (1976).

15. D. Tabor, Hardness of Metals. Clarendon Press, Oxford (1951).

16. S. K. R. Chowdhury and H. M. Pollock, Wear 66, 307 (1981).

17. T. S. Chow, Phys. Rev. Lett. 86, 4592 (2001).

18. T. S. Chow, J. Phys.: Condens. Matter 15, L83 (2003).

19. B. N. J. Persson, Phys. Rev. Lett. 87, 116101 (2001).

20. B. N. J. Persson, Phys. Rev. Lett. 88, 129601 (2002).

21. J. N. Israelachvili, in: Fundamentals of Friction: Macroscopic and Microscopic Processes, I. L. Singer and H. M. Pollock (Eds), p. 351. Kluwer, Dordrecht (1992).

22. K. L. Johnson, Tribol. Int. 31, 413 (1998).

23. K. L. Johnson, Contact Mechanics. Cambridge University Press, Cambridge (1985).

24. K. R. Morris, S. L. Nail, G. E. Peck, S. R. Byrn, U. J. Griesser, J. G. Stowell, S. J. Hwang and K. Park, Pharm. Sci. Technol. Today 1, 235 (1998).

25. R. Chiarello, V. Panella, J. Krim and C. Thompson, Phys. Rev. Lett. 67, 3408 (1991).

26. J. F. Archard, Tribol. Int. 7, 213 (1974). 